\title{
Projected properties of a family of triaxial mass models: High-order residuals
}

\author{
D. K. Chakraborty ${ }^{\star}$ and M. Das \\ School of Studies in Physics, Pt. Ravishankar Shukla University, Raipur 492 010, India \\ Received 27 May 2002 / Accepted 18 February 2003

\begin{abstract}
A family of triaxial mass models is described, for which the projected surface density can be calculated analytically, and can show ellipticity variations, isophote twists and high-order residuals. These can be compared with observations. These models are flattened versions of the $\gamma$-model with density $\rho(r) \propto r^{-\gamma}\left(b_{0}+r\right)^{\gamma-4}$ and the modified Hubble model with density profile $\rho(r) \propto\left(b_{0}^{2}+r^{2}\right)^{-3 / 2}$, and are constructed by addition of five spherical harmonic terms to the spherical models. The
\end{abstract} \\ potential of the models can be expressed in a simple form.
}

Key words. galaxies: photometry - galaxies: structure

\section{Introduction}

CCD photometry of elliptical galaxies shows that the isophotes are approximately elliptical, and ellipticity and position angle vary with radius. A triaxial mass model can produce variations in ellipticity and position angle in its projection. A spherical model $\rho(r)$, with density $\rho$ as a function of radius $r$, can be made triaxial by considering the expression for $\rho(r)$ and replacing $r^{2}$ by $m^{2}=x^{2}+y^{2} / p^{2}+z^{2} / q^{2}$, where $(x, y, z)$ are the usual Cartesian coordinates. The density is stratified on coaxial ellipsoids. In case the axial ratios $p$ and $q$ are constants, the projected density is stratified on similar and aligned ellipses (Stark 1977; Binney 1985). It was demonstrated by e.g. Madejsky \& Mollenhoff (1990) that models with equal density coaxial ellipsoidal shells with radially varying axial ratios can generate a variety of ellipticity and position angle profiles.

Triaxial mass models of an alternative form are $\rho(r, \theta, \phi)=$ $f(r)-g(r) Y_{2}^{0}(\theta)+h(r) Y_{2}^{2}(\theta, \phi)$, where $\rho$ is density in the usual spherical polar coordinates $(r, \theta, \phi), f(r)$ is a spherical mass distribution (which is usually a widely studied model), $g(r)$ and $h(r)$ are two suitably chosen radial functions, $Y_{2}^{0}=\frac{3}{2} \cos ^{2} \theta-\frac{1}{2}$ and $Y_{2}^{2}=3 \sin ^{2} \theta \cos 2 \phi$ are the usual spherical harmonics. These models also exhibit ellipticity variations and isophote twists in their projections (de Zeeuw \& Carollo 1996, hereafter ZC96, Chakraborty \& Thakur 2000, hereafter CT00). Such triaxial models with $f(r)$ as the spherical $\gamma$-models of Dehnen (1993), are presented in ZC96. Schwarzschild (1979) studied the triaxial model, wherein $f(r)$ is considered as the spherical modified Hubble model, in a numerical form. Later it was cast

Send offprint requests to: D. K. Chakraborty,

e-mail: ircrsu@sancharnet.in

* Also a Visiting Associate of IUCAA, Pune, India. into an analytical form by de Zeeuw \& Merritt (1983). The projected properties of such triaxial Hubble mass models are described in CT00. We shall refer to these models as $f g h$ models.

Although models with ellipticity variations and isophote twists have been discussed, high-order residuals in these models have not been adequately treated. A small value of $\sim-0.4 \%$ of the shape parameter has been reported in ZC96. Likewise, the isophotes at large radii are shown to be slightly boxy in CT00.

Rix \& White (1990) have discussed a photometric model, in which an exponential disk is embedded in the equatorial plane of an oblate spheroid of constant ellipticity, and have found that the isophotes are pointy. In a sense, the model is a two component model. Contopoulos \& Grosbol (1989) have found an orbital family which can lead to pointy isophotes. Thus, the model of Rix \& White is by no means an exclusive interpretation of ellipticals with pointy isophotes. Further, Binney \& Petrou (1985) have found that an overpopulation of a particular two dimensional subset of tube orbits can yield boxy isophotes.

Therefore, it will be worthwhile to examine density forms in a triaxial mass model which may produce non elliptical isophotes. For this investigation, we consider the $f g h$ models of ZC96 and CT00. The advantage of this approach is that the potential is known explicitly, and that the projected surface density can be calculated easily (and often, analytically). A disadvantage might be that the use of only the lower order spherical harmonic terms generally leads to models that become peanutshaped. The addition of higher order spherical harmonic terms leads to more nearly ellipsoidal shapes (Schwarzschild 1993), and "refines" the fgh models (ZC96).

Here we extend the studies of $f g h$ models by including higher-order spherical harmonic terms. We find that the models 
take more nearly ellipsoidal shapes, and further, the isophotes have high-order residuals. Depending upon the viewing directions, the isophotes are either boxy or pointy. Previous studies of the $f g h$ models lack this feature.

It was shown that the intrinsic shapes of triaxial mass models can be estimated by using photometric data (Thakur \& Chakraborty 2001). It is necessary to have a larger ensemble of models, before this method may be applied to elliptical galaxies. The present study of nearly ellipsoidal mass models is also a step towards this goal. Further, the profiles of high-order residuals of the models have some specific features. This may be used to select a galaxy which may be suitable for comparison with the models. For example, for the shape determination of elliptical galaxies, Statler (1994) had selected NGC 3379 and called it the "standard" elliptical galaxy, because, apart from factors like no sign of ripples or other fine structure, NGC 3379 has almost no isophote twist or ellipticity variation. The latter characteristics make NGC 3379 a suitable candidate for comparison with the models used by Statler, which also do not produce any isophote twist and ellipticity variation.

In Sect. 2, we describe the mass models and in Sect. 3, we present the projected properties. Section 4 is devoted to results and a discussion.

\section{Mass model}

\subsection{A potential-density pair}

First, we consider a potential of the form

$V_{2}(r, \theta, \phi)=u_{1}(r)+u_{2}(r) Y_{l}^{m}(\theta, \phi)$,

where $u_{1}(r)$ and $u_{2}(r)$ are two radial functions, and $Y_{l}^{m}(\theta, \phi)=$ $P_{l}^{m}(\cos \theta) \cos m \phi$. Here, $P_{l}^{m}(\cos \theta)$ are the usual associated Legendre polynomials, and we have considered only the real part of the spherical harmonics $Y_{l}^{m}$. Then, Poisson's equation gives the mass density $\rho_{2}$ of the form

$4 \pi G \rho_{2}=\frac{\mathrm{d}^{2} u_{1}}{\mathrm{~d} r^{2}}+\frac{2}{r} \frac{\mathrm{d} u_{1}}{\mathrm{~d} r}+g_{2}(r) Y_{l}^{m}$,

where $g_{2}(r)$ is the contribution to density corresponding to $u_{2}(r)$ in potential, and is given by

$g_{2}(r)=\frac{\mathrm{d}^{2} u_{2}}{\mathrm{~d} r^{2}}+\frac{2}{r} \frac{\mathrm{d} u_{2}}{\mathrm{~d} r}-\frac{l(l+1)}{r^{2}} u_{2}$.

In the above, we have used the differential equation

$$
\frac{1}{\sin \theta} \frac{\partial}{\partial \theta}\left(\sin \theta \frac{\partial P_{l}^{m}}{\partial \theta}\right)+\left[l(l+1)-\frac{m^{2}}{\sin ^{2} \theta}\right] P_{l}^{m}=0
$$

satisfied by $P_{l}^{m}$. For $l=2$ expression (3) reduces to the form used by Schwarzschild (1979).

\subsection{A family of triaxial mass models}

We now consider a potential $V(r, \theta, \phi)$ of the form

$$
\begin{aligned}
V(r, \theta, \phi)= & V_{1}(r, \theta, \phi)+v_{1}(r)\left[Y_{4}^{0}(\theta)-\alpha Y_{4}^{4}(\theta, \phi)\right] \\
& +w_{1} Y_{4}^{2}(\theta, \phi),
\end{aligned}
$$

where

$V_{1}(r, \theta, \phi)=u(r)-v(r) Y_{2}^{0}(\theta)+w(r) Y_{2}^{2}(\theta, \phi)$.

In the above, $u(r), v(r), w(r), v_{1}(r)$ and $w_{1}(r)$ are five radial functions, $Y_{2}^{0}=\frac{3}{2} \cos ^{2} \theta-\frac{1}{2}, Y_{2}^{2}=3 \sin ^{2} \theta \cos 2 \phi, Y_{4}^{0}=$ $\frac{1}{8}\left(35 \cos ^{4} \theta-30 \cos ^{2} \theta+3\right), Y_{4}^{2}=\frac{15}{2}\left(7 \cos ^{2} \theta-1\right) \sin ^{2} \theta \cos 2 \phi$, $Y_{4}^{4}=105 \sin ^{4} \theta \cos 4 \phi$ and $\alpha$ is a parameter.

Applying Eqs. (1)-(3), we obtain the associated density $\rho(r, \theta, \phi)$, given by

$$
\begin{aligned}
\rho(r, \theta, \phi)= & \rho_{1}(r, \theta, \phi)+s(r)\left[Y_{4}^{0}(\theta)-\alpha Y_{4}^{4}(\theta, \phi)\right] \\
& +t(r) Y_{4}^{2}(\theta, \phi),
\end{aligned}
$$

where

$\rho_{1}(r, \theta, \phi)=f(r)-g(r) Y_{2}^{0}(\theta)+h(r) Y_{2}^{2}(\theta, \phi)$.

The radial functions $f(r), g(r), h(r), s(r)$ and $t(r)$ are obtained from $u(r), v(r), w(r), v_{1}(r)$ and $w_{1}(r)$, respectively, by using relations which can be read from Eqs. (1)-(3). The density $\rho_{1}(r, \theta, \phi)$ and the associated potential $V_{1}(r, \theta, \phi)$ define the $f g h$ models.

It was realized by Schwarzschild (1979) that while the first term in (8) is spherical, the second term containing $Y_{2}^{0}$ shortens the $z$-axis and lengthens the $x$ and $y$ axes equally. The third term containing $Y_{2}^{2}$ lengthens the $x$-axis and shortens the $y$-axis. This gives rise to a triaxial figure with the major axis along the $x$-coordinate, the median one along the $y$-coordinate and the minor one along the $z$-coordinate. The additional, second and third terms in (7) just reverse the above effects, provided $\alpha>3 /(8 \times 105)$. We take $\alpha=0.01$ for the present study.

We now consider two specific examples of models. We consider an extension of the models in ZC96, according to the scheme proposed above, and refer to these as models $A$. We consider a potential of the form (5) and take $u(r)$ of the form

$u(r)= \begin{cases}\frac{M G}{b_{0}} \ln \frac{r}{b_{0}+r}, & \text { for } \gamma=2, \\ \frac{M G}{(2-\gamma) b_{0}}\left[\left(\frac{r}{b_{0}+r}\right)^{2-\gamma}-1\right], & \text { for } \gamma \neq 2,\end{cases}$

with $0 \leq \gamma<3$. In the above, $M$ is the mass of the model and $b_{0}$ is the scale length. The radial functions $v(r)$ and $w(r)$, chosen in ZC96, have the form $r^{2-\gamma} /(1+r)^{4-\gamma}$. Then the corresponding radial functions in the expression of density, as obtained by using Eq. (3), turn out to be positive at all $r$. Following this, we consider a form $r^{n-\gamma} /(1+r)^{m-\gamma}$, both for $v_{1}(r)$ and $w_{1}(r)$. The corresponding radial functions in the expression of density are positive at all $r$, if $n=4$ and $m \leq 9$. The lower limit of $m$ is restricted so that $v_{1}(r)$ and $w_{1}(r)$ decrease faster than $v(r)$ and $w(r)$, at large radii. These considerations lead us to the following choices. We choose

$$
\begin{aligned}
& v(r)=-M G \frac{b_{1} r^{2-\gamma}}{\left(b_{2}+r\right)^{4-\gamma}}, \\
& w(r)=-M G \frac{b_{3} r^{2-\gamma}}{\left(b_{4}+r\right)^{4-\gamma}}, \\
& v_{1}(r)=-M G \frac{c_{1}^{6}}{b_{0}^{3}} \frac{r^{4-\gamma}}{\left(c_{2}+r\right)^{8-\gamma}}
\end{aligned}
$$



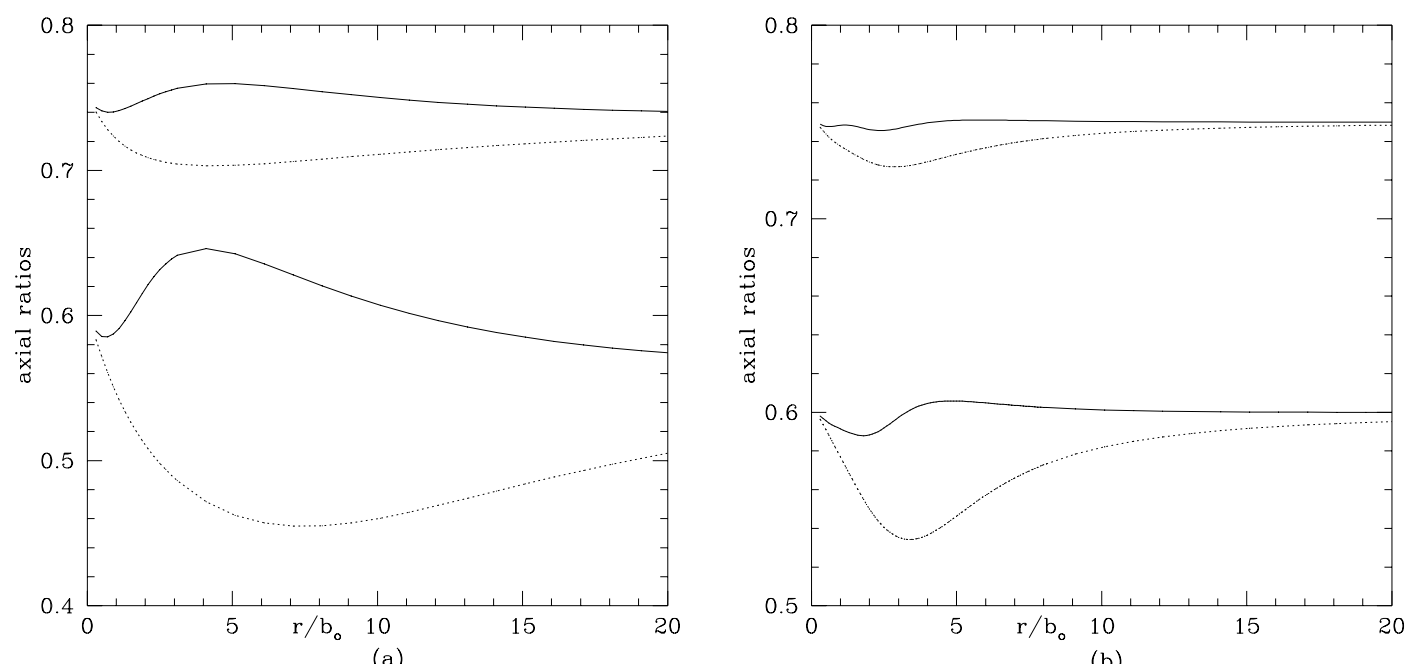

(b)

Fig. 1. Axial ratios of constant- $\rho_{1}$ surfaces (dotted lines), constant $\rho$ surfaces (solid lines) with $p=0.75, q=0.6$. Frame a) presents the model $A$ with $\gamma=1.5$ and frame b) presents the model $B$.

and

$w_{1}(r)=-M G \frac{c_{3}^{6}}{b_{0}^{3}} \frac{r^{4-\gamma}}{\left(c_{4}+r\right)^{8-\gamma}}$.

The associated density $\rho(r, \theta, \phi)$ is given by (7) and (8), where

$f(r)=\frac{M b_{0}}{4 \pi} \frac{(3-\gamma)}{r^{\gamma}\left(b_{0}+r\right)^{4-\gamma}}$,

$g(r)=\frac{M b_{1}}{4 \pi} \frac{\left[4 r^{2}+4(6-\gamma) r b_{2}+\gamma(5-\gamma) b_{2}^{2}\right]}{r^{\gamma}\left(b_{2}+r\right)^{6-\gamma}}$,

$h(r)=\frac{M b_{3}}{4 \pi} \frac{\left[4 r^{2}+4(6-\gamma) r b_{4}+\gamma(5-\gamma) b_{4}^{2}\right]}{r^{\gamma}\left(b_{4}+r\right)^{6-\gamma}}$,

$s(r)=\frac{M c_{1}^{6}}{4 \pi b_{0}^{3}} \frac{r^{2}\left[8 r^{2}+8(10-\gamma) r c_{2}+\gamma(9-\gamma) c_{2}^{2}\right]}{r^{\gamma}\left(c_{2}+r\right)^{10-\gamma}}$

and

$t(r)=\frac{M c_{3}^{6}}{4 \pi b_{0}^{3}} \frac{r^{2}\left[8 r^{2}+8(10-\gamma) r c_{4}+\gamma(9-\gamma) c_{4}^{2}\right]}{r^{\gamma}\left(c_{4}+r\right)^{10-\gamma}}$.

In the above, $b_{1}, b_{2}, b_{3}, b_{4}, c_{1}, c_{2}, c_{3}$ and $c_{4}$ are constants.

As an another example of triaxial models, we take $u(r)$ to be the potential of the spherical modified Hubble model, defined by the choice

$u(r)=-\frac{M G}{r}\left\{\ln \left[\frac{r}{b_{0}}+\sqrt{1+\frac{r^{2}}{b_{0}^{2}}}\right]\right\}$,

and consider its triaxial generalization of the form (5). These models will be called models $B$. de Zeeuw \& Merritt (1983) had adopted a form $r^{2} /\left(1+r^{2}\right)^{3 / 2}$, both for $v(r)$ and $w(r)$. Following this, we consider a form $r^{n} /\left(1+r^{2}\right)^{m}$, both for $v_{1}(r)$ and $w_{1}(r)$. We find that the corresponding radial functions in the density are also positive if $n=4$ and $m \leq 9 / 2$. We choose

$v(r)=-M G \frac{b_{1}^{3}}{b_{0}^{3}} \frac{r^{2}}{\left(b_{2}^{2}+r^{2}\right)^{3 / 2}}$,

$$
\begin{aligned}
& w(r)=-M G \frac{b_{3}^{3}}{b_{0}^{3}} \frac{r^{2}}{\left(b_{4}^{2}+r^{2}\right)^{3 / 2}}, \\
& v_{1}(r)=-M G \frac{c_{1}^{5}}{b_{0}^{3}} \frac{r^{4}}{\left(c_{2}^{2}+r^{2}\right)^{7 / 2}}, \\
& w_{1}(r)=-M G \frac{c_{3}^{5}}{b_{0}^{3}} \frac{r^{4}}{\left(c_{4}^{2}+r^{2}\right)^{7 / 2}} .
\end{aligned}
$$

The mass model is defined by (7) and (8) with the radial functions

$f(r)=\frac{M}{4 \pi} \frac{1}{\left(b_{0}^{2}+r^{2}\right)^{3 / 2}}$,

$g(r)=\frac{3 M}{4 \pi} \frac{b_{1}^{3}}{b_{0}^{3}} \frac{2 r^{4}+7 b_{2}^{2} r^{2}}{\left(b_{2}^{2}+r^{2}\right)^{7 / 2}}$,

$h(r)=\frac{3 M}{4 \pi} \frac{b_{3}^{3}}{b_{0}^{3}} \frac{2 r^{4}+7 b_{4}^{2} r^{2}}{\left(b_{4}^{2}+r^{2}\right)^{7 / 2}}$,

$s(r)=\frac{7 M}{4 \pi} \frac{c_{1}^{5}}{b_{0}^{3}} \frac{2 r^{6}+11 c_{2}^{2} r^{4}}{\left(c_{2}^{2}+r^{2}\right)^{11 / 2}}$

and

$t(r)=\frac{7 M}{4 \pi} \frac{c_{3}^{5}}{b_{0}^{3}} \frac{2 r^{6}+11 c_{4}^{2} r^{4}}{\left(c_{4}^{2}+r^{2}\right)^{11 / 2}}$.

The four ratios $\frac{b_{1}}{b_{0}}, \ldots, \frac{b_{4}}{b_{0}}$ in the mass models $A$ and $B$ can be expressed in terms of the axial ratios $\left(p_{\infty}, q_{\infty}\right)$ and $\left(p_{0}, q_{0}\right)$ of the density distribution $\rho$ at (asymptotically) large and at (asymptotically) small radii (see ZC96 \& CT00), where the surfaces of constant density are approximately ellipsoidal.

We find that even when $p_{0}=p_{\infty}=p$ and $q_{0}=q_{\infty}=q$ are considered, the axial ratios of constant $-\rho_{1}$ surfaces fall short of $p$ and $q$, at intermediate radii (see Fig. 1). Confining ourselves to the case where $p_{0}=p_{\infty}=p$ and $q_{0}=q_{\infty}=q$, we set the values of $c_{1}, \ldots, c_{4}$ such that at a large (finite) and at a small (finite) major axis lengths, the axial ratios of constant- $\rho$ surfaces become close to $p$ and $q$ (Appendix A). 

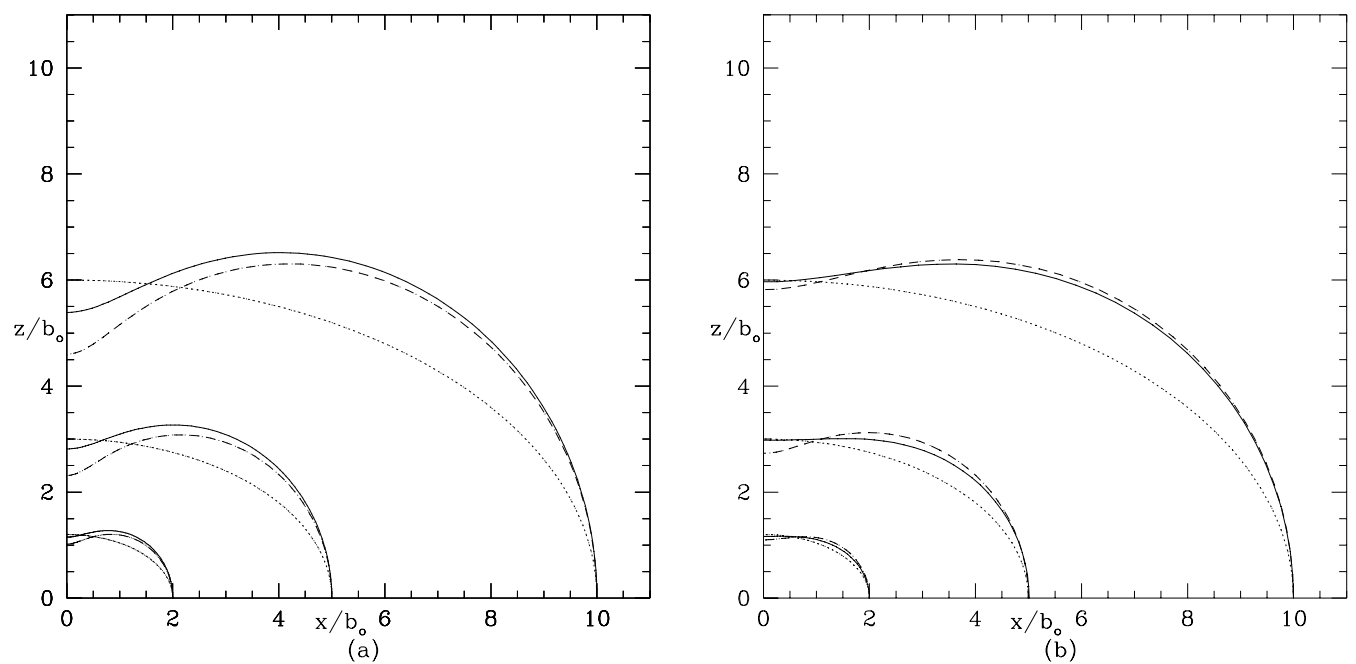

Fig. 2. Sections of the constant- $\rho_{1}$ surfaces (dashed lines), constant- $\rho$ surfaces (solid lines) and the ellipsoids (dotted lines) with $p=0.75$, $q=0.6$ with different choices of the major axis lengths $a$, on the $x-z$ plane. Frame a) presents the model $A$ with $\gamma=1.5$ and frame b) presents the model $B$.

We have adopted a form (7) of density distribution and a procedure to determine $c 1, \ldots, c 4$. The resulting density figure is nearly ellipsoidal: serious dimples of the $f g h$ model have almost disappeared (see Fig. 2). The method adopted here is different from that adopted by Schwarzschild (1993), who used a form equivalent to a 10 term development in spherical harmonics.

\section{Projected properties}

The form (7) of the density of the models allows a straightforward calculation of the projected surface density $\Sigma$, for any viewing direction. We choose coordinates $\left(x^{\prime}, y^{\prime}, z^{\prime}\right)$ with the $z^{\prime}$-axis along the line of sight and with the $x^{\prime}$-axis in the $(x, y)$ plane. Let $\left(\theta^{\prime}, \phi^{\prime}\right)$ be the standard spherical coordinates of the line of sight (de Zeeuw \& Franx 1989) and $(R, \Theta)$ be the polar coordinates in the $\left(x^{\prime}, y^{\prime}\right)$ plane. The projected surface density $\Sigma(R, \Theta)$ is then given by

$\Sigma(R, \Theta)=\Sigma_{1}(R, \Theta)+P_{4}(R) \cos 4 \Theta+P_{5}(R) \sin 4 \Theta$,

where

$\Sigma_{1}(R, \Theta)=P_{0}(R)-P_{2}(R) \cos 2 \Theta-P_{3}(R) \sin 2 \Theta$.

The radial functions $P_{0}, \ldots, P_{5}$ are expressed in terms of the integrals $S_{1}(R), S_{2}(R), S_{3}(R)$, given by

$S_{1}(R)=\int_{R}^{\infty} \frac{r s(r) \mathrm{d} r}{\sqrt{r^{2}-R^{2}}}$,
$S_{2}(R)=R^{2} \int_{R}^{\infty} \frac{s(r) \mathrm{d} r}{r \sqrt{r^{2}-R^{2}}}$,
$S_{3}(R)=R^{4} \int_{R}^{\infty} \frac{s(r) \mathrm{d} r}{r^{3} \sqrt{r^{2}-R^{2}}}$,

and similar integrals $T_{1}, T_{2}, T_{3}, G_{1}, G_{2}, H_{1}, H_{2}$ and $F_{1}$ in terms of the functions $t(r), g(r), h(r)$ and $f(r)$, respectively. The actual forms of these functions $P_{0}, \ldots, P_{5}$ are presented in Appendix B. The integrals needed in $P_{0}, \ldots, P_{5}$ are calculated analytically for models $B$, and are presented in Appendix C. This can be regarded as an added advantage of considering the modified Hubble model. For models $A$, these integrals are calculated by numerical quadrature of the formulae given in Appendix D. These can also be expressed in terms of elementary functions for integer $\gamma$ (Appendix E).

Although the potential $V_{1}(r, \theta, \phi)$ and the associated density $\rho_{1}(r, \theta, \phi)$ are the same as in the $f g h$ models studied by earlier workers, we note that $\Sigma_{1}(R, \Theta)$ is now different from the projected density $\Sigma_{f g h}(R, \Theta)$ of the $f g h$ models. The additional terms with radial functions $s(r)$ and $t(r)$, which are included in the present study, also contribute to $\Sigma_{1}(R, \Theta)$. However, the contributions from these additional terms are small compared to those from the terms with radial functions $f(r), g(r)$ and $h(r)$. The functional form of $\Sigma_{1}(R, \Theta)$ is same as that of $\Sigma_{f g h}(R, \Theta)$. We follow the derivations made in ZC96 and define the position angle $\Theta_{\star \Sigma_{1}}$ of $\Sigma_{1}$ by

$\Theta_{\star \Sigma_{1}}=\frac{1}{2} \tan ^{-1} \frac{P_{3}}{P_{2}}$

and the axial ratio $b / a$ of $\Sigma_{1}$ by

$$
\begin{aligned}
& P_{0}(a)-P_{2}(a) \cos 2 \Theta_{\star \Sigma_{1}}-P_{3}(a) \sin 2 \Theta_{\star \Sigma_{1}}=P_{0}(b) \\
& \quad-P_{2}(b) \cos 2\left(\Theta_{\star \Sigma_{1}}-\pi / 2\right)-P_{3}(b) \sin 2\left(\Theta_{\star \Sigma_{1}}-\pi / 2\right) .
\end{aligned}
$$

Relation (34) gives the position angle of the major axis, provided

$P_{3} \sin 2 \Theta_{\star \Sigma_{1}}<0$.

The terms $P_{4}$ and $P_{5}$ are related to high-order residuals, on constant- $\Sigma_{1}$ contour, as can be seen by rewriting (29) as

$$
\begin{aligned}
\Sigma(R, \Theta)= & \Sigma_{1}(R, \Theta)+B_{4 \Sigma_{1}} \cos 4\left(\Theta-\Theta_{\star \Sigma_{1}}\right) \\
& +A_{4 \Sigma_{1}} \sin 4\left(\Theta-\Theta_{\star \Sigma_{1}}\right),
\end{aligned}
$$

where

$B_{4 \Sigma_{1}}=P_{4} \cos 4 \Theta_{\star \Sigma_{1}}+P_{5} \sin 4 \Theta_{\star \Sigma_{1}}$,

$A_{4 \Sigma_{1}}=P_{5} \cos 4 \Theta_{\star \Sigma_{1}}-P_{4} \sin 4 \Theta_{\star \Sigma_{1}}$. 
Table 1. Profiles of the isophotal shape parameter $B_{4}$, position angle $\Theta_{*}$ of the major axis and the ellipticity $\epsilon$ of the best-fitting ellipses, all as a function of the semimajor axis length $a$, expressed in terms of the scale length $b_{0}$. These can be compared with the corresponding parameters $B_{4 \Sigma_{1}}, \Theta_{*_{\Sigma_{1}}}$ and $\epsilon_{\Sigma_{1}}$ of the constant $-\Sigma_{1}$ contours.

\begin{tabular}{ccccccc|c}
\hline \hline$a / b_{0}$ & $B_{4}$ & $B_{4 \Sigma_{1}}$ & $\Theta_{*}$ & $\Theta_{* \Sigma_{1}}$ & $\epsilon$ & $\epsilon_{\Sigma_{1}}$ & comments \\
\hline 1 & 0.005 & 0.017 & $90.0^{\circ}$ & $88.5^{\circ}$ & 0.20 & 0.22 & Model $A$ \\
2 & 0.023 & 0.032 & $90.1^{\circ}$ & $88.7^{\circ}$ & 0.22 & 0.22 & $p=0.9, q=0.7$ \\
3 & 0.033 & 0.035 & $90.9^{\circ}$ & $90.4^{\circ}$ & 0.22 & 0.22 & $\gamma=1.5$ \\
4 & 0.033 & 0.034 & $91.4^{\circ}$ & $91.0^{\circ}$ & 0.21 & 0.21 & $\theta^{\prime}=80^{\circ}$ \\
5 & 0.030 & 0.030 & $91.9^{\circ}$ & $91.4^{\circ}$ & 0.21 & 0.21 & $\phi^{\prime}=20^{\circ}$ \\
6 & 0.025 & 0.026 & $92.2^{\circ}$ & $91.7^{\circ}$ & 0.20 & 0.21 & \\
\hline 1 & -0.019 & -0.019 & $-29.8^{\circ}$ & $-29.6^{\circ}$ & 0.07 & 0.07 & Model $B$ \\
2 & -0.021 & -0.015 & $-34.0^{\circ}$ & $-34.5^{\circ}$ & 0.09 & 0.08 & $p=0.9, q=0.7$ \\
3 & -0.014 & -0.009 & $-35.1^{\circ}$ & $-35.6^{\circ}$ & 0.08 & 0.08 & $\theta^{\prime}=30^{\circ}$ \\
4 & -0.010 & -0.006 & $-35.4^{\circ}$ & $-35.7^{\circ}$ & 0.08 & 0.08 & $\phi^{\prime}=20^{\circ}$ \\
6 & -0.006 & -0.003 & $-35.5^{\circ}$ & $-35.7^{\circ}$ & 0.08 & 0.08 & \\
8 & -0.004 & -0.002 & $-35.6^{\circ}$ & $-35.6^{\circ}$ & 0.08 & 0.08 & \\
12 & -0.003 & -0.001 & $-35.6^{\circ}$ & $-35.6^{\circ}$ & 0.08 & 0.08 & \\
20 & -0.002 & -0.001 & $-35.6^{\circ}$ & $-35.6^{\circ}$ & 0.07 & 0.07 & \\
\hline
\end{tabular}

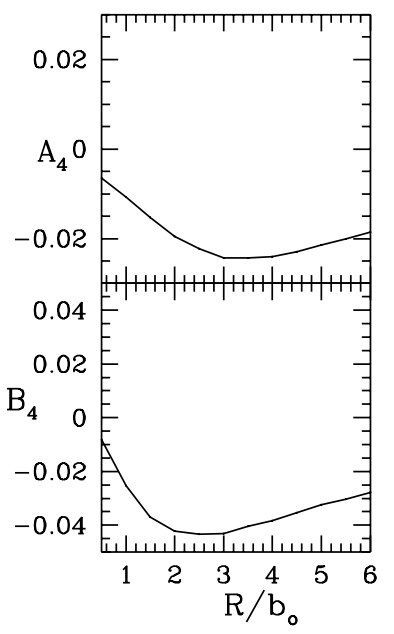

(a)

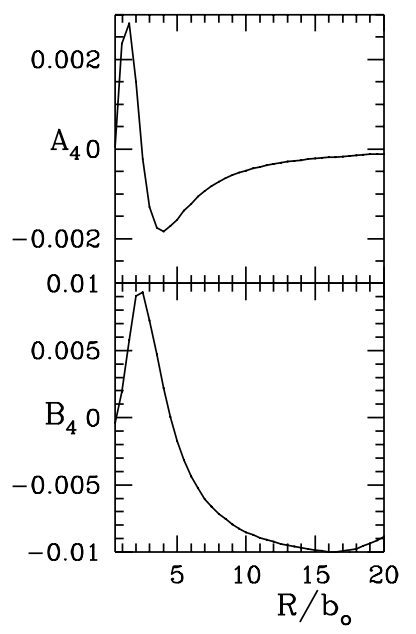

(b)
Fig. 3. Radial profiles of $B_{4}$ and $A_{4}$ for models with $p=0.9, q=0.7$. Frame a) presents model $A$ with $\gamma=1.5, \theta^{\prime}=30^{\circ}, \phi^{\prime}=20^{\circ}$ and frame b) presents model $B$ with $\theta^{\prime}=80^{\circ}, \phi^{\prime}=20^{\circ}$.

\section{Results and discussion}

We have obtained the projected density of a family of triaxial mass models which are nearly ellipsoidal. Although the complexity of the problem has led us to some long expressions, the numerical evaluation of these to obtain the projected parameters, in terms of different choices of the model parameters and viewing angles, is straightforward.

Figure 1 shows the axial ratios of constant density surfaces of the models $A$ and $B$. We find that the axial ratios of constant $\rho_{1}$ surfaces at intermediate radii, vary considerably from their values at asymptotic radii. These variations are relatively small for the constant- $\rho$ surfaces. For the model $A$ with $p=0.75, q=0.6, \gamma=1.5$, the maximum relative change in the axial ratios of constant $-\rho_{1}$ surfaces is $\sim 25.0 \%$, which drops to $\sim 8.3 \%$ for constant $-\rho$ surfaces. For the same choice of $p$ and $q$, the model $B$ exhibits the maximum relative change in the axial ratios of $\sim 11.6 \%$ for constant $-\rho_{1}$ surfaces and $\sim 1.6 \%$ for constant $-\rho$ surfaces. For the rounder models, e.g. with $p=0.9, q=0.7$ these variations are relatively small.

In Fig. 2, we present the sections of constant density surfaces of the models and the ellipsoids, with the same $p$ and $q$, in the $(x-z)$ plane. While the constant- $\rho_{1}$ is dimpled (peanutshaped), the constant $\rho$ is relatively smooth and closer to the ellipsoid.

In Sect. 3, we have defined $B_{4 \Sigma_{1}}$ and $A_{4 \Sigma_{1}}$, which are related to high-order residuals on constant- $\Sigma_{1}$ contours. The surface photometry data of elliptical galaxies are obtained by considering variations around the best-fitting ellipse. In order to compare the model with data, we use the standard routines of ellipse-fitting based on the algorithm of Jedrzejewski (1987), and obtain the parameters of the best-fitting ellipses on $\Sigma$ and the high-order residuals $A_{3}, B_{3}, A_{4}, B_{4}$ on the ellipses. We find that $B_{4}$ is positive when viewed almost along the $(x-y)$ plane and it is negative when viewed at narrow angles with respect to the $\pm z$ directions. At intermediate viewing angles, $B_{4}$ is partly positive and partly negative. $A_{4}$ is small compared to $B_{4}$, and the residuals $A_{3}$ and $B_{3}$ are negligible.

Figure 3 and Table 1 present the radial profiles of $B_{4}$ and $A_{4}$ to a radial distance of nearly four times the effective radius of each model. Table 1 also presents the profiles of basic parameters, namely, the position angle $\Theta_{*}$ of the major axis and the ellipticity $\epsilon$ of the best-fitting ellipse, and the corresponding parameters $\Theta_{* \Sigma_{1}}$ and $\epsilon_{\Sigma_{1}}$ of the constant $-\Sigma_{1}$ contour. It is seen that the basic parameters of the best-fitting ellipses are very close to those of the constant- $\Sigma_{1}$ contours. Finally, we find the centres of the best-fitting ellipses coincide with the centre of $\Sigma$.

Models $A$ and $B$ appear to be either boxy or pointy, depending upon the viewing direction. Regions of these viewing directions are shown in Fig. 4 . We find that the model $A$ exhibits three distinct regions of viewing angles producing boxy (region 1), partly boxy and partly pointy (region 2) and pointy 


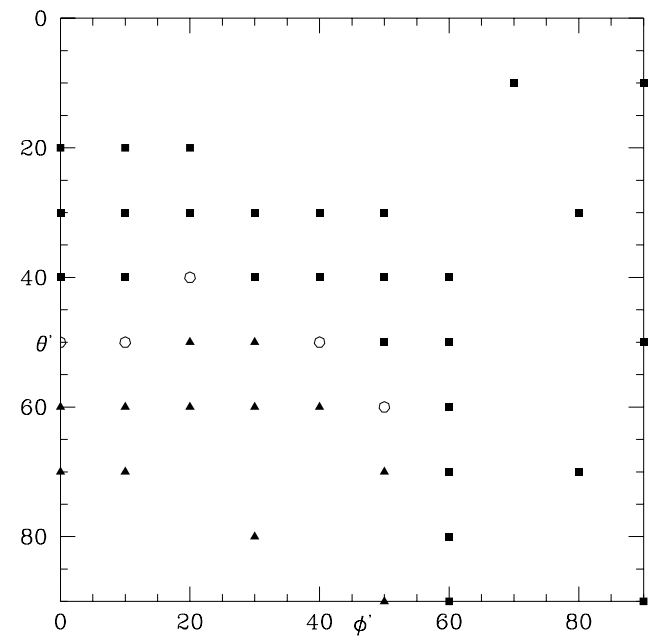

(a)

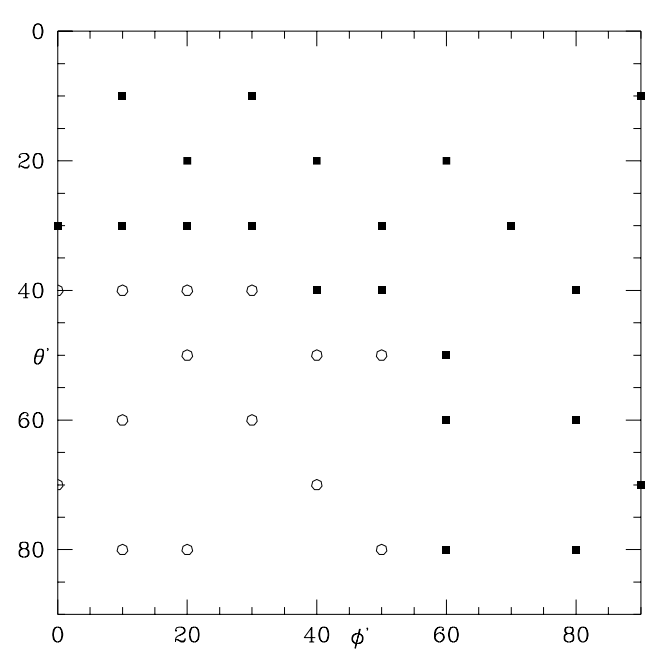

(b)

Fig. 4. Viewing directions for boxy and pointy isophotes for models, with $p=0.9, q=0.7$. Points indicate the viewing angles at which the ellipse-fitting tasks were performed. Filled squares, filled triangles and open circles indicate the viewing directions for boxy, pointy and partly pointy isophotes, respectively. Frame a) presents the model $A$ with $\gamma=1.5$ and frame b) presents the model $B$.

Table 2. $<(s+t) /(g+h)>$ and the maximum variations in axial ratios.

\begin{tabular}{ccccccc}
\hline \hline Model & $p$ & $q$ & $\gamma$ & \multicolumn{2}{c}{ Maximum variation in axial ratios } & $<(s+t) /(g+h)>$ \\
& & & & constant $\rho_{1}$ surfaces & constant- $\rho$ surfaces & \\
\hline$A$ & 0.9 & 0.7 & 1.5 & $15.6 \%$ & $6.3 \%$ & 0.37 \\
& 0.75 & 0.6 & 1.5 & $25.0 \%$ & $8.3 \%$ & 0.30 \\
$B$ & 0.9 & 0.7 & - & $8.5 \%$ & $1.4 \%$ & 0.055 \\
& 0.75 & 0.6 & - & $11.6 \%$ & $1.6 \%$ & 0.046 \\
\hline
\end{tabular}

(region 3) isophotes. On the other hand the model $B$ shows regions 1 and 2 only.

To understand this feature, we perform the following numerical experiment. We obtain the residuals on the best-fitting ellipses on $\Sigma_{1}$. The isophotes of $\Sigma_{1}$ are found to be boxy in all the viewing directions. It follows that the pointy isophotes of $\Sigma$ arise only from the $s$ and $t$ terms in $\rho$ (see Eqs. (B.2) and (B.3)). A measure of the contributions from the $s$ and $t$ terms in $\rho$, may be the average of the ratio $(s+t) /(g+h)$. Further, note that the terms $s$ and $t$ were added to reduce the variations in the axial ratios of the $f g h$ models (cf. Fig. 1). Therefore, it is expected that the contributions from the $s$ and $t$ terms should be related to the variations in the axial ratios. Table 1 presents these parameters. It is seen that $\langle(s+t) /(g+h)>$ is smaller in model $B$ than in model $A$. Consistently, the variation in axial ratios in model $B$ is smaller than in model $A$.

The isophotes of elliptical galaxies with disks have been found to exhibit similar behavior (Scorza \& Bender 1995; Rix \& White 1990). Using a model with an exponential disk embedded in an oblate spheroid, Rix and White have found that $B_{4}$ is positive, which is the photometric signature of the disk, only when the angle $i$ of the disk from the line of sight is less than $\cos ^{-1}(0.6)$. In the mass models presented here, the radial functions $s(r)$ and $t(r)$ decay faster than the other radial functions, both at small and at large radii. The terms in $\rho$ with these radial functions, appear to mimic a disc in projection.
The analytical formulation gives us some insight. We find that the fourth order residuals $B_{4 \Sigma_{1}}$ and $A_{4 \Sigma_{1}}$ arise, only from the fourth order spherical harmonic terms in $\rho$, and no third order or higher order residuals are present in our analytical formulation. Our results of the ellipse-fitting algorithm are consistent with these analytical findings. We also note that the projected density has the symmetry $\Sigma(R, \Theta)=\Sigma(R, \Theta+\pi)$. The radial coordinate of the points on an ellipse, with centre as origin, also has this symmetry. We may anticipate that the centre of the best-fitting ellipse should coincide with the center of $\Sigma$. This is found to be true.

Although, $B_{4 \Sigma_{1}}$ can not be compared with the observations, it can still be used for a qualitative estimate of the variation in the high-order residual with the viewing direction. Only for the rounder models with $p=0.9, q=0.7, B_{4 \Sigma_{1}}$ is close to $B_{4}$ (see Table 1). However, the ellipticity and position angle of the best-fitting ellipse are quite close to the ellipticity and position angle of $\Sigma_{1}$, for any semi major axis length, even for the flatter models with $p=0.75, q=0.6$.

Acknowledgements. DKC, a Visiting Associate of the InterUniversity Centre for Astronomy and Astrophysics (IUCAA), Pune, India, and MD express their sincere thanks to IUCAA for providing local hospitality and support during IUCAA visits. Partial support is also provided through the CSIR project grant No. 03(0807)/97/EMR-II, which is gratefully acknowledged. We are very grateful to the anonymous referee for his critical comments and useful suggestions, which helped us to improve the paper enormously. 


\section{Appendix A}

We expand $s(r)$ and $t(r)$ at large and at small $r$ and retain the terms of highest orders only, in $\rho$. Requiring that at a large and at a small major axis lengths, denoted $a_{1}$ and $a_{\mathrm{s}}$, respectively, axial ratios of constant- $\rho$ surfaces are same as $p$ and $q$, we obtain

$c_{1}^{n}=\frac{a_{1}^{n} q^{n}}{d_{1}} \frac{\beta\left\{\rho_{1 x_{1}}-\rho_{1 z_{1}}+p^{n}\left(\rho_{1 y_{1}}-\rho_{1 z_{1}}\right)\right\}}{\left\{1+p^{n}-2 a_{0} q^{n}\right\}}$,

$c_{3}^{n}=\frac{a_{1}^{n} \beta}{d_{2}\left\{1+p^{n}-2 a_{0} q^{n}\right\}}\left\{\rho_{1 x_{1}}\left(p^{n}-a_{0} q^{n}\right)-\rho_{1 y_{1}} p^{n}\left(1-a_{0} q^{n}\right)\right.$

$\left.+\rho_{1 z_{1}} q^{n} a_{0}\left(1-p^{n}\right)\right\}$

where $\rho_{1 x_{1}}=\rho_{1}\left(a_{1}, 0,0\right), \rho_{1 y_{1}}=\rho_{1}\left(0, a_{1} p, 0\right), \rho_{1 z_{1}}=$ $\rho_{1}\left(0,0, a_{1} q\right), \beta=4 \pi b_{0}^{3} / M$ and $a_{0}=\frac{3}{8}-105 \alpha$, and $\left(n, d_{1}, d_{2}\right)$ are $(6,8,60)$ for models $A$ and $(5,14,105)$ for models $B$. On the other hand at $a_{\mathrm{s}}$, we obtain

$$
\begin{aligned}
\frac{c_{1}^{n}}{c_{2}^{m+n}}= & \frac{\beta}{d_{1} a_{\mathrm{s}}^{m}} \frac{\left\{\rho_{1 x_{\mathrm{s}}} p^{m}+\rho_{1 y_{\mathrm{s}}}-\rho_{1 z_{\mathrm{s}}}\left(1+p^{m}\right)\right\}}{\left\{q^{m}\left(1+p^{m}\right)-2 a_{0} p^{m}\right\}}, \\
\frac{c_{3}^{n}}{c_{4}^{m+n}}= & \frac{2 \beta}{d_{2} a_{\mathrm{s}}^{m}\left\{q^{m}\left(1+p^{m}\right)-2 a_{0} p^{m}\right\}} \\
& \times\left\{\rho_{1 x_{\mathrm{s}}}\left(q^{m}-a_{0} p^{m}\right)-\rho_{1 y_{\mathrm{s}}}\left(q^{m}-a_{0}\right)\right. \\
& \left.-\rho_{1 z_{\mathrm{s}}} a_{0}\left(1-p^{m}\right)\right\},
\end{aligned}
$$

where $\rho_{1 x_{\mathrm{s}}}=\rho_{1}\left(a_{\mathrm{s}}, 0,0\right), \rho_{1 y_{\mathrm{s}}}=\rho_{1}\left(0, a_{\mathrm{s}} p, 0\right), \rho_{1 z_{\mathrm{s}}}=$ $\rho_{1}\left(0,0, a_{\mathrm{s}} q\right)$, and $\left(n, m, d_{1}, d_{2}\right)$ are $(6,2-\gamma, \gamma(9-\gamma), 15 \gamma(9-\gamma))$ for models $A$ (with $\gamma \neq 0$ ) and are $(5,4,77,1155)$ for models $B$. We take $a_{1}=20 b_{0}$ and $a_{\mathrm{s}}=0.5 b_{0}$ and find that the terms, which are dropped while obtaining (A1)-(A4), are negligible.

\section{Appendix B}

Integrating $\rho$ along the line of sight, we obtain

$$
\begin{aligned}
\Sigma= & E_{1}+E_{2} \sin ^{2} \Theta+E_{3} \cos ^{2} \Theta+E_{4} \sin \Theta \cos \Theta \\
& +E_{5} \sin ^{4} \Theta+E_{6} \cos ^{4} \Theta+E_{7} \sin ^{2} \Theta \cos ^{2} \Theta \\
& +E_{8} \cos \Theta \sin ^{3} \Theta+E_{9} \sin \Theta \cos ^{3} \Theta,
\end{aligned}
$$

where

$$
\begin{aligned}
E_{1}= & 2 F_{1}+G_{1}+\frac{3}{4} S_{1}+3 \cos ^{2} \theta^{\prime}\left\{G_{2}-G_{1}+\frac{5}{2}\left(S_{2}-S_{1}\right)\right\} \\
& +\sin ^{2} \theta^{\prime} \cos 2 \phi^{\prime}\left\{6\left(H_{1}-H_{2}\right)+15\left(T_{2}-T_{1}\right)\right\} \\
& +105 \sin ^{2} \theta^{\prime} \cos ^{2} \theta^{\prime} \cos 2 \phi^{\prime}\left\{T_{3}-2 T_{2}+T_{1}\right\} \\
& -\left(210 \alpha \sin ^{4} \theta^{\prime} \cos 4 \phi^{\prime}-\frac{35}{4} \cos ^{4} \theta^{\prime}\right) \\
& \times\left\{S_{3}-2 S_{2}+S_{1}\right\} \\
E_{2}= & -\frac{3}{2} \sin ^{2} \theta^{\prime}\left\{2 G_{2}+5 S_{2}\right\}+3 \cos ^{2} \theta^{\prime} \cos 2 \phi^{\prime}\left\{2 H_{2}-5 T_{2}\right\} \\
& +\frac{105}{2} \sin ^{2} \theta^{\prime} \cos ^{2} \theta^{\prime}\left(24 \alpha \cos 4 \phi^{\prime}-1\right) \\
& \times\left\{S_{3}-S_{2}\right\}-105\left(\cos ^{4} \theta^{\prime}+\sin ^{4} \theta^{\prime}-4 \sin ^{2} \theta^{\prime}\right. \\
& \left.\times \cos ^{2} \theta^{\prime}\right) \cos 2 \phi^{\prime}\left\{T_{3}-T_{2}\right\},
\end{aligned}
$$

$E_{3}=-3 \cos 2 \phi^{\prime}\left\{2 H_{2}-5 T_{2}\right\}-1260 \alpha \sin ^{2} \theta^{\prime} \cos 4 \phi^{\prime}$ $\times\left\{S_{3}-S_{2}\right\}+105 \cos ^{2} \theta^{\prime} \cos 2 \phi^{\prime}\left\{T_{3}-T_{2}\right\}$,

$E_{4}=6 \sin 2 \phi^{\prime} \cos \theta^{\prime}\left\{2 H_{2}-5 T_{2}\right\}+5040 \alpha \sin 2 \phi^{\prime}$ $\times \cos 2 \phi^{\prime} \sin ^{2} \theta^{\prime} \cos \theta^{\prime}\left\{S_{3}-S_{2}\right\}-210 \sin 2 \phi^{\prime}$ $\times \cos \theta^{\prime}\left(\cos ^{2} \theta^{\prime}-2 \sin ^{2} \theta^{\prime}\right)\left\{T_{3}-T_{2}\right\}$,

$E_{5}=\frac{35}{4} \sin ^{4} \theta^{\prime} S_{3}-210 \alpha \cos ^{4} \theta^{\prime} \cos 4 \phi^{\prime} S_{3}$ $+105 \sin ^{2} \theta^{\prime} \cos ^{2} \theta^{\prime} \cos 2 \phi^{\prime} T_{3}$,

$E_{6}=-210 \alpha \cos 4 \phi^{\prime} S_{3}$,

$E_{7}=1260 \alpha \cos 4 \phi^{\prime} \cos ^{2} \theta^{\prime} S_{3}-105 \cos 2 \phi^{\prime} \sin ^{2} \theta^{\prime} T_{3}$,

$E_{8}=-1680 \alpha \cos 2 \phi^{\prime} \sin 2 \phi^{\prime} \cos ^{3} \theta^{\prime} S_{3}$ $+210 \sin 2 \phi^{\prime} \sin ^{2} \theta^{\prime} \cos \theta^{\prime} T_{3}$,

$E_{9}=1680 \alpha \sin 2 \phi^{\prime} \cos 2 \phi^{\prime} \cos \theta^{\prime} S_{3}$,

which can be written in the form (29). We have

$P_{0}=E_{1}+\frac{1}{2}\left(E_{2}+E_{3}\right)+\frac{1}{8}\left(3 E_{5}+3 E_{6}+E_{7}\right)$,

$P_{2}=-\frac{1}{2}\left(E_{2}-E_{3}+E_{5}-E_{6}\right)$,

$P_{3}=\frac{1}{4}\left(2 E_{4}+E_{8}+E_{9}\right)$,

$P_{4}=\frac{1}{8}\left(E_{5}+E_{6}-E_{7}\right)$,

$P_{5}=-\frac{1}{8}\left(E_{8}-E_{9}\right)$.

\section{Appendix C}

The radial functions $S_{1}, S_{2}, S_{3}, T_{1}, T_{2}, T_{3}, H_{1}, H_{2}, G_{1}, G_{2}$ and $F_{1}$ in $P_{0} \ldots P_{5}$ can be evaluated analytically. The integrands can be cast as integral powers of $\cos \theta$ which can be integrated easily. Writting

$I_{1}=R^{6} t_{1}+3 R^{4} t_{2}+3 R^{2} t_{3}+t_{4}$,

$I_{2}=R^{4} t_{1}+2 R^{2} t_{2}+t_{3}$,

$I_{3}=R^{2} t_{1}+t_{2}$

and

$I_{4}=t_{1}$,

where $t_{1}=\frac{128}{315} \frac{1}{\left(c_{2}^{2}+R^{2}\right)^{5}}, t_{2}=\frac{16}{315} \frac{1}{\left(c_{2}^{2}+R^{2}\right)^{4}}, t_{3}=\frac{8}{315} \frac{1}{\left(c_{2}^{2}+R^{2}\right)^{3}}$, $t_{4}=\frac{2}{63} \frac{1}{\left(c_{2}^{2}+R^{2}\right)^{2}}$,

we have

$S_{1}(R)=\frac{M}{4 \pi b_{0}^{3}}\left(14 c_{1}^{5} I_{1}+77 c_{1}^{5} c_{2}^{2} I_{2}\right)$,

$S_{2}(R)=\frac{M R^{2}}{4 \pi b_{0}^{3}}\left(14 c_{1}^{5} I_{2}+77 c_{1}^{5} c_{2}^{2} I_{3}\right)$,

$S_{3}(R)=\frac{M R^{4}}{4 \pi b_{0}^{3}}\left(14 c_{1}^{5} I_{3}+77 c_{1}^{5} c_{2}^{2} I_{4}\right)$.

$T_{1}, \ldots T_{3}$ can be obtained from $(C 2)$ by replacing $\left(c_{1}, c_{3}\right)$ by $\left(c_{2}, c_{4}\right)$. Expressions for $G_{1}, G_{2}, H_{1}, H_{2}$ and $F_{1}$ have been reported earlier (CT00). These can also be rewritten in forms which have similar appearances, as above. 
Table E.1. The functions $S_{1}, S_{2}, S_{3}$ for special values of $\gamma$, expressed in terms of the elementary functions $W_{n}(x)$.

\begin{tabular}{|c|c|}
\hline$\gamma$ & Function \\
\hline \multirow[t]{3}{*}{0} & $\begin{aligned} S_{1}(R) & =\frac{M c_{1}^{6}}{\pi b_{0}^{3} c_{2}^{5}}\left[2 W_{5}\left(R / c_{2}\right)+10 W_{6}\left(R / c_{2}\right)-60 W_{7}\left(R / c_{2}\right)+100 W_{8}\left(R / c_{2}\right)\right. \\
& \left.-70 W_{9}\left(R / c_{2}\right)+18 W_{10}\left(R / c_{2}\right)\right]\end{aligned}$ \\
\hline & $S_{2}(R)=\frac{M c_{1}^{6} R^{2}}{\pi b_{0}^{3} c_{2}^{7}}\left[2 W_{7}\left(R / c_{2}\right)+14 W_{8}\left(R / c_{2}\right)-34 W_{9}\left(R / c_{2}\right)+18 W_{10}\left(R / c_{2}\right)\right]$ \\
\hline & $S_{3}(R)=\frac{M c_{1}^{6} R^{4}}{\pi b_{0}^{3} c_{2}^{9}}\left[2 W_{9}\left(R / c_{2}\right)+18 W_{10}\left(R / c_{2}\right)\right]$ \\
\hline \multirow[t]{3}{*}{1} & $\begin{aligned} S_{1}(R) & =\frac{M c_{1}^{6}}{\pi b_{0}^{3} c_{2}^{5}}\left[2 W_{5}\left(R / c_{2}\right)+10 W_{6}\left(R / c_{2}\right)-40 W_{7}\left(R / c_{2}\right)+42 W_{8}\left(R / c_{2}\right)\right. \\
& \left.-14 W_{9}\left(R / c_{2}\right)\right]\end{aligned}$ \\
\hline & $S_{2}(R)=\frac{M c_{1}^{6} R^{2}}{\pi b_{0}^{3} c_{2}^{7}}\left[2 W_{7}\left(R / c_{2}\right)+14 W_{8}\left(R / c_{2}\right)-14 W_{9}\left(R / c_{2}\right)\right]$ \\
\hline & $\begin{aligned} S_{3}(R) & =\frac{M c_{1}^{6} R^{4}}{\pi b_{0}^{3} c_{2}^{9}}\left[\frac{2 c_{2}^{2}}{R^{2}}-2 W_{2}\left(R / c_{2}\right)-4 W_{3}\left(R / c_{2}\right)-6 W_{4}\left(R / c_{2}\right)-8 W_{5}\left(R / c_{2}\right)\right. \\
& \left.-10 W_{6}\left(R / c_{2}\right)-12 W_{7}\left(R / c_{2}\right)-14 W_{8}\left(R / c_{2}\right)-14 W_{9}\left(R / c_{2}\right)\right]\end{aligned}$ \\
\hline \multirow[t]{3}{*}{2} & $S_{1}(R)=\frac{M c_{1}^{6}}{2 \pi b_{0}^{3} c_{2}^{5}}\left[4 W_{5}\left(R / c_{2}\right)+20 W_{6}\left(R / c_{2}\right)-45 W_{7}\left(R / c_{2}\right)+21 W_{8}\left(R / c_{2}\right)\right]$ \\
\hline & $\begin{array}{l}S_{2}(R)=\frac{M c_{1}^{6} R^{2}}{2 \pi b_{0}^{3} c_{2}^{7}}\left[\frac{7 \pi c_{2}}{2 R}-7 W_{1}\left(R / c_{2}\right)-7 W_{2}\left(R / c_{2}\right)-7 W_{3}\left(R / c_{2}\right)-7 W_{4}\left(R / c_{2}\right)\right. \\
\left.-7 W_{5}\left(R / c_{2}\right)-7 W_{6}\left(R / c_{2}\right)-3 W_{7}\left(R / c_{2}\right)+21 W_{8}\left(R / c_{2}\right)\right]\end{array}$ \\
\hline & $\begin{aligned} S_{3}(R) & =\frac{M c_{1}^{6} R^{4}}{2 \pi b_{0}^{3} c_{2}^{9}}\left[\frac{7}{4} \frac{\pi c_{2}^{3}}{R^{3}}-\frac{24 c_{2}^{2}}{R^{2}}+24 W_{2}\left(R / c_{2}\right)+41 W_{3}\left(R / c_{2}\right)+51 W_{4}\left(R / c_{2}\right)\right. \\
& \left.+54 W_{5}\left(R / c_{2}\right)+50 W_{6}\left(R / c_{2}\right)+39 W_{7}\left(R / c_{2}\right)+21 W_{8}\left(R / c_{2}\right)\right]\end{aligned}$ \\
\hline
\end{tabular}

\section{Appendix D}

The formulae given in (31)-(33) are not very useful for numerical computations because of the infinite integration intervals and singularities of the integrands. Following Dehnen (1993) and Thakur \& Chakraborty (2001), we put

$\tau=\sqrt{\frac{r}{r+c_{2}}(1+\sigma)-\sigma}$,

where

$\sigma=\frac{R}{c_{2}}$

to obtain

$$
\begin{aligned}
S_{1}(R)= & \frac{M c_{1}^{6}}{2 \pi b_{0}^{3} c_{2}^{5}(1+\sigma)^{19 / 2-\gamma}} \\
& \times \int_{0}^{1} f(\tau)\left(\sigma+\tau^{2}\right)^{3-\gamma}\left(1-\tau^{2}\right)^{4} \mathrm{~d} \tau, \\
S_{2}(R)= & \frac{M c_{1}^{6} R^{2}}{2 \pi b_{0}^{3} c_{2}^{7}(1+\sigma)^{19 / 2-\gamma}} \\
& \times \int_{0}^{1} f(\tau)\left(\sigma+\tau^{2}\right)^{1-\gamma}\left(1-\tau^{2}\right)^{6} \mathrm{~d} \tau,
\end{aligned}
$$

$S_{3}(R)=\frac{M c_{1}^{6} R^{4}}{2 \pi b_{0}^{3} c_{2}^{9}(1+\sigma)^{19 / 2-\gamma}} \int_{0}^{1} \frac{f(\tau)\left(1-\tau^{2}\right)^{8} \mathrm{~d} \tau}{\left(\sigma+\tau^{2}\right)^{1+\tau}}$

where

$$
\begin{aligned}
f(\tau)= & \frac{1}{\sqrt{2 \sigma+\tau^{2}(1-\sigma)}} \\
& \times\left\{8\left(\sigma+\tau^{2}\right)^{2}+8(10-\gamma)\left(\sigma+\tau^{2}\right)\left(1-\tau^{2}\right)\right. \\
& \left.+\gamma(9-\gamma)\left(1-\tau^{2}\right)^{2}\right\} .
\end{aligned}
$$

The integrads are free from the singularities and intervals of integrations are finite. These can be computed easily by using standard routines of numerical quadrature.

Similar forms for integrals $F_{1}, G_{1}$ and $G_{2}$ are reported in Thakur \& Chakraborty (2001). 


\section{Appendix E}

For integer $\gamma$, the integral $F_{1}, G_{1}, G_{2}, S_{1}, S_{2}$ and $S_{3}$ can be written in terms of functions $W_{n}(x)$, which are defined as follows (ZC96):

$W_{1}(x)= \begin{cases}\frac{1}{\sqrt{1-x^{2}}} \ln \frac{1+\sqrt{1-x^{2}}}{x}, & (0 \leq x<1), \\ 1, & (x=1), \\ \frac{1}{\sqrt{x^{2}-1}} \arccos \frac{1}{x}, & (x>1),\end{cases}$

$W_{2}(x)=\frac{W_{1}(x)-1}{1-x^{2}}$

and

$$
\begin{aligned}
W_{n}(x)= & \frac{1}{(n-1)\left(1-x^{2}\right)}\left\{(2 n-3) W_{n-1}(x)\right. \\
& \left.-(n-2) W_{n-2}(x)\right\}, \quad(n>2) .
\end{aligned}
$$

In Table E.1, we present the functions $S_{1}, S_{2}$ and $S_{3}$, expressed in terms of $W_{n}(x)$. Functions $F_{1}, G_{1}, G_{2}$ in terms of $W_{n}(x)$ are reported in ZC96.

\section{References}

Binney, J. 1985, MNRAS, 212, 767

Binney, J., \& Petrou, M. 1985, MNRAS, 214, 449

Chakraborty, D. K., \& Thakur, P. 2000, MNRAS, 318, 1273 (CT00)

Contopoulos, G., \& Grosbol, P. 1989, Astr. Ap. Rev., 1, 261

de Zeeuw, P. T., \& Carollo, C. M. 1996, MNRAS, 281, 1333 (ZC96)

de Zeeuw, P. T., \& Franx, M. 1989, ApJ, 343, 617

de Zeeuw, T., \& Merritt, D. 1983, ApJ, 267, 571

Dehnen, W. 1993, MNRAS, 265, 250

Jedrzejewski, R. I. 1987, MNRAS, 226, 747

Madejsky, R., \& Mollenhoff, C. 1990, A\&A, 234, 119

Rix, H. W., \& White, S. D. M. 1990, ApJ, 362, 52

Schwarzschild, M. 1979, ApJ, 232, 236

Schwarzschild, M. 1993, ApJ, 409, 563

Scorza, C., \& Bender, R. 1995, A\&A, 293, 20

Stark, A. A. 1977, ApJ, 213, 368

Statler, T. S. 1994, AJ, 108, 111

(E.3) Thakur, P., \& Chakraborty, D. K. 2001, MNRAS, 328, 330 